\title{
Classification of Cardiac Arrhythmias Using Feed Forward Neural Network
}

\author{
$\nabla^{1}$ R R Karhe, ${ }^{2}$ Dr. S N Kale \\ ${ }^{1}$ Department of Electronics \& Telecommunication Shri Gulabrao Deokar College of Engineering Jalgaon, \\ Maharashtra, India \\ rajeshkarhe@yahoo.com \\ ${ }^{2}$ Department of Applied Science, Sant Gadge Baba Amravati University, Amravati, Maharashtra, India \\ sujatankale@rediffmail.com
}

Received: 04th August 2020, Accepted: 29th September 2020, Published: 31st October 2020

\begin{abstract}
Arrhythmias are abnormal rhythms of heart. An irreparable damage is caused due to arrhythmias like Ventricular Tachycardia (VT) and Ventricular Fibrillation (VF). Timely detection of such fatal arrhythmias is vital for the successive application of defibrillation therapies. Heart diseases are diagnosed using different types of tests. Electrocardiography (ECG) testing is one of most efficient and non-invasive method among all. The defined algorithms used for detection and classification of arrhythmias are based on surface Electrocardiogram analysis. However, in this research, an algorithm based on shape statistical and spectral kurtosis features is presented. In this algorithm, shape statistical features along with spectral kurtosis features are applied to the classifier to classify and detect the type of arrhythmia. Various performance measure like accuracy, execution time and comparison of various performance functions like MSE, SSE, SAE, MAE along with training functions like Trainrp, Trainbr, Trainscg, Trainlm is done in order to identify the most excellent neural network models. The results shows an average accuracy of $90.57 \%$ with Feed Forward neural network (FFNN) as the most excellent neural network (NN) for classification of cardiac arrhythmia. The ultimate objective of this algorithm is to contribute as a coherent heart diagnostic and arrhythmia detection tool.
\end{abstract}

\section{Keywords}

Arrhythmias, ECG, Shape Statistical Features, Spectral Kurtosis Features, Neural Networks

\section{Introduction}

In 2008 about 17.3 million people died from heart disease, according to World Health Organization (WHO) estimation and this number of death will increase upto 23.3 million in 2030 . Hence heart diseases become very casual disease. To diminish the number of death and extend the duration quality of life, a new method for early detection of heart health, can be adopted.

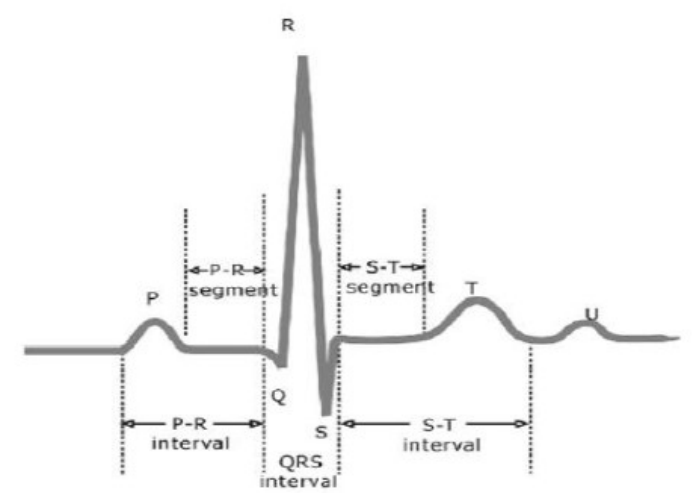

Figure 1: ECG Signal

The normal Electrocardiogram (ECG) signal is as shown in figure 1. ECG imitates by the pulsing action of heart determinant by heart contraction and reflection and has been widely used for examination of heart posture. ECG signal poised of aspect known as $\mathrm{P}$ wave, QRS complex, T wave and $\mathrm{U}$ wave. Any abnormality with respect to such aspects indicates a heart arrhythmia. RR interval is the continuation within consecutive standard ECG beat, which measures the heart rate. Heart Rate $=[60 / \mathrm{RR}$ interval $]$ beats per min. Normally heart rate is $60-100$ beats per min for human. The departure in heart rate from the standard rate point out the abnormal working of heart which is intimation of heart arrhythmia.

Various approaches are introduced in advances to achieve computerized arrhythmia diagnosis system based on the attribute of the ECG signal. Since the ECG diagnosis is a computer based function so furnishing of most outstanding features of ECG is very important by which the accurate diagnosis is feasible. Many authors 
approaches on attention on various characteristic like preprocessing, feature extraction, and learning techniques. The focus of this research paper is to expand computerized diagnosis method for diagnosis and classification of heart arrhythmia by examining the ECG signal. To study the ECG signal is very simple, it is repeatable signal. The study concerns with different kinds of arrhythmias databases from MIT-BIH data namely: Standard Arrhythmia ECG Data, CU Ventricular Tachyarrhythmia Database, Supraventricular Arrhythmia Database, and Polysomnographic Arrhythmias database. The performance is evaluated for different neural network architectures.

This paperwork is put in order as follows: in section 2 the literature review of arrhythmias detection and classification is described. In section 3 details about the database. Methodology is described in section 4 . In section 5 describe the investigational study. Conclusion is described in section 6 .

\section{Existing Systems}

The Literature Review emphasizes on work done by researchers on analyzing different methods for prediction or classification of cardiac arrhythmia.

Cuiwei Li et al. [3] have implemented wavelets transform to extract QRS complex .Bert. Ume Kohler et al. [4] have presented a software for QRS detection algorithms in cardiological devices. M.Sabarimalai Manikandan et al.[5] have proposed an automated logic to detect ' $R$ ' peaks. Jiapu Pan deve et .al [6] have developed an real time algorithm for diagnosis of QRS complex in view of slope, width information and amplitude in the ECG signal .V. S. Chouhan et al.[7] have reported derivative based approach for QRS detection E. B. Mazomenos et al. [8] have implemented TDMG algorithm to extract PQRST signals.Philip de Chazal et al. [9] have developed an method for evaluating features based on Morphology, RR intervals, heartbeat intervals and classified by statistical classifier model for heartbeat classification. A. K. M. Fazlul Haque et al.[10] have presented a method to extract small variation features in ECG with using time-varying morphological characteristics by using signal processing method. Kutlu and Kuntalp et al. [11] have proposed an algorithm based on high order statistics for arrhythmia recognition system. Hari Mohan Rai et al. [12] have suggested analysis of ECG in which morphological, wavelet features are implemented to distinguish into abnormal and normal classes. Niwas SI et al. [13] have used the ANN for classification of heartbeats of ECG, in which they accurately classify the heartbeat of ECG signals and evaluating features based on Spectral Entropy, RR intervals, heartbeat intervals. The result shows classification of nine types of arrhythmia and normal heart beat. A Dallali et al.[14] have used ANN (artificial neural network) and WT (Wavelet Transformation) for classification of ECG signals in which HRV (Heart Rate Variability) is one of the important feature which is taken into consideration while classification of ECGs. Shivajirao M. Jadhav et al. [15] have used UCI database for ECG signal analysis in order to test and train by three ANN models by backpropagation algorithm along with momentum learning rule in order to analyze cardiac arrhythmias. Maedeh kiani sarkaleh [16] et al have implemented DWT transform for processing ECG signal to extract features and multilayer perception (MLP) neural network to carry out classification function the simulation result shows 96.5\%.accurcy. A. T. Sayad et al [17] have implemented thirteen attributes for diagnosing heart diseases. In this methodology Multi-layer Perceptron Neural Network along with training algorithm as Back-propagation is used for prediction of risk of heart disease. BOUSSAA Mohamed et al.[18] implemented Harr like features and ANN on real time ECG images. Siva A et al. [19] have implemented DWT for extraction of ECG features. Gandham Sreedevi et al. [20] have used LMBPNN (Levenberg-Marquardt Back Propagation Neural Network) for extraction of ECG from normal as well as abnormal ECG. Eyhab Al-Masriet al.[21] have developed an neural network based algorithm in order to extract morphological features . Yeldos A. Altay et al. [22] have designed a neural network clustering algorithm which is based on machine learning

Database

The MIT BIH arrhythmia database gives Physiological ECG signal which is implemented in this work. Here four types of arrhythmia database are considered for classification of arrhythmia (Total 175 ECG database signals) .such as Standard Arrhythmia ECG Data of 45 samples, CU Ventricular Tachyarrhythmia database of 35samples, Supraventricular Arrhythmia Database of 77 samples, Polysomnographic Arrhythmia Database of 18 samples. The MIT BIH arrhythmias data base has number of ECG recording and all these Signals are sampled $250 \mathrm{~Hz}$.

\section{Methodology}

The method for arrhythmia detection is implemented in MATLAB 2013a. Figure 2 shows the MATLAB based GUI for the proposed system. 


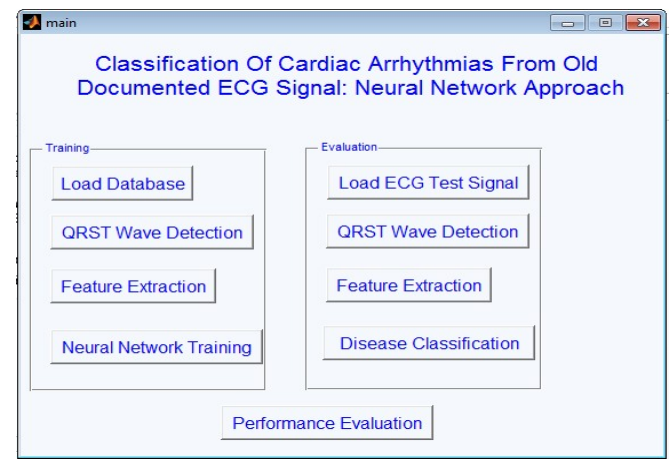

Figure 2: MATLAB Based System for Classification of Arrhythmias

The flowchart of the proposed methodology for detection of arrhythmia data from normal data is shown in figure 3. The overall technique is distributed into three main parts QRST wave identification and then feature extraction and feature classification by ANN. For detection of QRST waves the raw ECG signal is provided as an input. For finding the QRST peaks of ECG, there should be preprocessing of the original ECG signal will done. The next step is feature extraction that identifies attributes of the original signal. Finally this processed signal is to classify into two class which is normal and arrhythmia class.

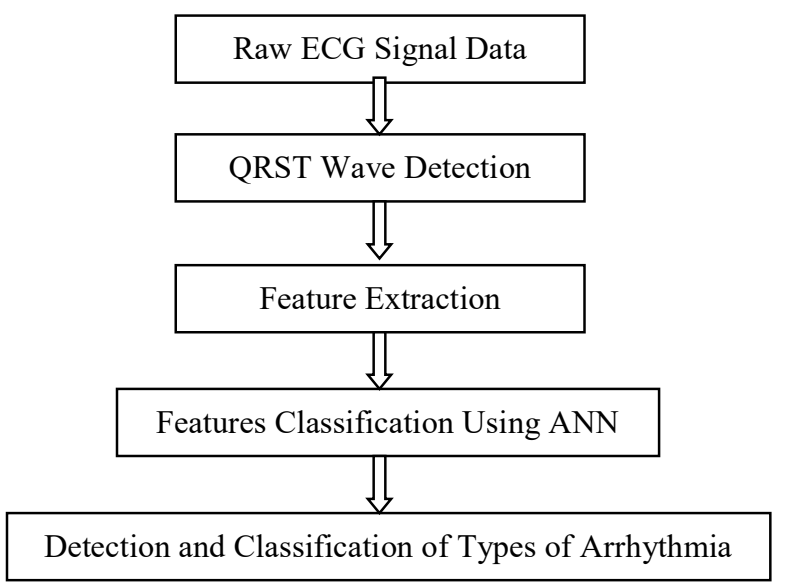

A. QRST wave detection

Figure 3: Algorithm

The process of QRST wave detection is to identify different peaks in an ECG signal. With the help of high pass filtering and low pass filtering it is possible to eliminate noise and baseline wander respectively.

The steps for QRST wave detection are as follows

1. Noise cancelation (Filtering) includes cutoff low frequency for elimination of baseline wander, cutoff frequency to remove high frequency noise, cut off based on fs, bandpass filtering

2. Go in for state 1 for recognizing $R$ wave and locate ' $R$ ' by finding highest Peak and also locate ' $Q$ '

3. Go in for state 2 for $\mathrm{S}$ wave detection to check if signal fall under the threshold to see for $\mathrm{S}$ wave

4. Go in for state 3 for $\mathrm{S}$ wave detection by identifying slope changes.

5. Go in for state 4 for $\mathrm{T}$ wave detection and identify the signal fall under mean.

6. Go in for state 5 in which if $\mathrm{T}$ wave is present it gets detected.

B. Features Extraction

Using data obtained from MIT-BIH the statistical analysis and classification among normal and abnormal conditions is performed using MATLAB. The following features like mean, standard deviation, Skewness, Kurtosis, Peak to Peak, RMS, CrestFactor, Shape Factor, Impulse Factor, Margin Factor, Energy and Spectral Kurtosis related features like SKMean, SKStd, SKSkewness, SKKurtosis are extracted.

C. Neural Network

The research work is implemented for the classification of ECG signal using Neural Network with different hidden neurons and initial weights assumed to be zero. The parameters are implemented for classification of features using neural network are Input Vector, and Target Vector .The overall classification is performed using input vector and target vector with neural network. In this research work we have considered four architectures of the neural network for classification of types of arrhythmias they are as follows: Feedforwardnet, Patternnet, 
Cascadednet, Layercnet (Recurrent Neural Network). The performance is evaluated for various numbers of hidden neurons.

\section{Result and Discussions}

To evaluate the performance of the proposed system, we have considered five measures as, Accuracy, precision, recall, F measure and execution time. These parameters are defined using four measures True Positive (TP), True Negative (TN), False Positive (FP), and False Negative (FN). All of these parameters are explained in the following ways.

Accuracy -Accuracy is a standard of measurement for evaluating classification models.

$\begin{aligned} \text { Accuracy }= & \frac{\text { Numberof correctpredictions }}{\text { Total number of predictions }} \\ & =\mathrm{TP}+\mathrm{TN} / \mathrm{TP}+\mathrm{TN}+\mathrm{FP}+\mathrm{FN}\end{aligned}$

Precision - It is the proportion of accurately predicted positive detection inside the total estimated positive detection.

Precision $=\mathrm{TP} / \mathrm{TP}+\mathrm{FP}$.

Recall (Sensitivity) - Recall is the proportion of accurately predicted positive detection to actual positive detection

Recall $=\mathrm{TP} / \mathrm{TP}+\mathrm{FN}$.

F score - it is the harmonic mean of Recall and Precision.

F Score $=2 *(($ Recall $*$ Precision $) /($ Recall + Precision $))$

Table 1: Performance of Neural Network for various number of Hidden Neurons

\begin{tabular}{|c|c|c|c|c|c|}
\hline \multicolumn{6}{|c|}{ Accuracy } \\
\hline No of Hidden Neurons & 5 & 10 & 15 & 20 & 25 \\
\hline FFNN & 86.57 & 91.71 & 90.57 & 92.02 & 92.06 \\
\hline Patternnet & 78.28 & 90.01 & 77.71 & 80.28 & 84.02 \\
\hline CascadedForwardNet & 78.85 & 76.85 & 94.07 & 87.71 & 93.42 \\
\hline LayrecNet & 85.71 & 85.71 & 85.71 & 85.71 & 85.71 \\
\hline \multicolumn{6}{|c|}{ Precision } \\
\hline No of Hidden Neurons & 5 & 10 & 15 & 20 & 25 \\
\hline FFNN & 77.33 & 82.25 & 84.81 & 84.45 & 84.98 \\
\hline Patternnet & 78.85 & 80.13 & 62.54 & 56.87 & 70.35 \\
\hline CascadedForwardNet & 79.24 & 72.57 & 89.27 & 83.86 & 87.46 \\
\hline LayrecNet & 80.58 & 80.58 & 80.58 & 80.58 & 80.58 \\
\hline \multicolumn{6}{|c|}{ Recall } \\
\hline No of Hidden Neurons & 5 & 10 & 15 & 20 & 25 \\
\hline FFNN & 68.51 & 85.05 & 78.04 & 86.94 & 89.07 \\
\hline Patternnet & 56.15 & 75.48 & 50.13 & 60.49 & 82.2 \\
\hline CascadedForwardNet & 55.41 & 57.68 & 91.5 & 69.18 & 90.69 \\
\hline LayrecNet & 68.63 & 68.63 & 68.63 & 68.63 & 68.63 \\
\hline \multicolumn{6}{|c|}{ F Measure } \\
\hline No of Hidden Neurons & 5 & 10 & 15 & 20 & 25 \\
\hline FFNN & 28.05 & 26.42 & 34.28 & 49.41 & 66.03 \\
\hline Patternnet & 49.41 & 77.42 & 46.42 & 56.46 & 72.48 \\
\hline CascadedForwardNet & 56.28 & 48.94 & 89.56 & 70.19 & 88.15 \\
\hline LayrecNet & 69.76 & 69.76 & 69.76 & 69.76 & 69.76 \\
\hline \multicolumn{6}{|c|}{ Execution Time (sec) } \\
\hline No of Hidden Neurons & 5 & 10 & 15 & 20 & 25 \\
\hline FFNN & 6.5405 & 0.5087 & 0.5624 & 0.6806 & 0.6221 \\
\hline Patternnet & 0.3813 & 0.5431 & 0.4521 & 0.5145 & 0.5995 \\
\hline CascadedForwardNet & 6.1404 & 0.3661 & 0.5507 & 0.5159 & 0.7025 \\
\hline LayrecNet & 1.4516 & 0.9586 & 1.1581 & 1.425 & 1.8456 \\
\hline
\end{tabular}

As depicted in table 1 'Patternnet' neural network requires less execution time. But the accuracy of FFNN architecture is more for detection and classification of arrhythmias.

As shown in figure 3, FFNN gives best accuracy for the arrhythmia classification and the execution time for all $\mathrm{NN}$ is shown in figure 4 


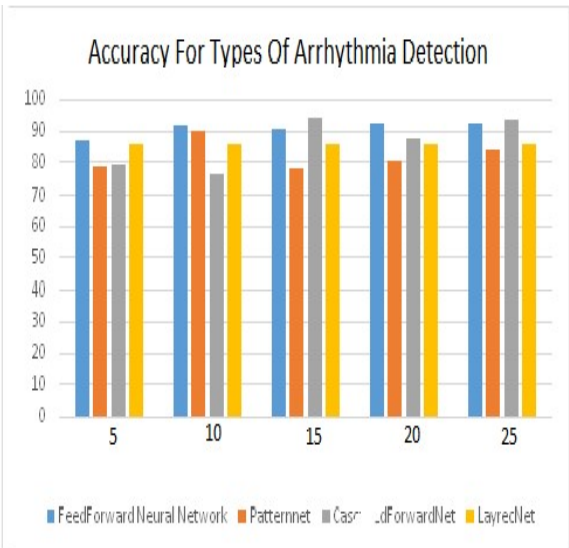

Figure 4: Classification Accuracy

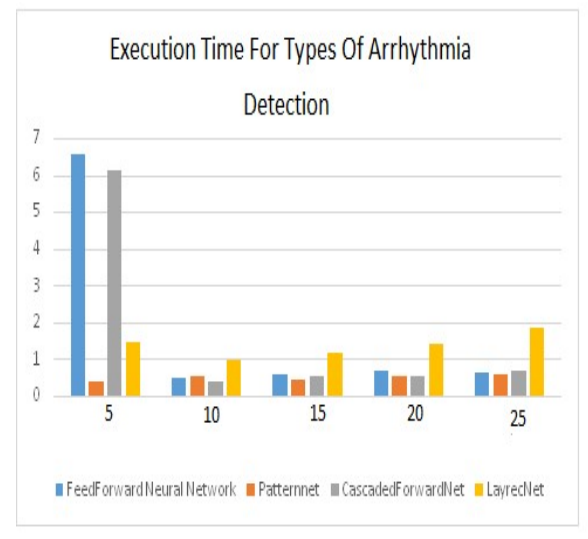

Figure 5: Execution Time

Evaluating the Performance of Types of Arrhythmia Detection for Various Training Functions:The performance of neural networks for various training functions is evaluated for FFNN architectures.

Table 2: Performance for various Training function

\begin{tabular}{|l|c|c|}
\hline \multirow{2}{*}{ Training Functions } & \multicolumn{2}{|c|}{ Feed Forward Net } \\
\cline { 2 - 3 } & Avg. Accuracy & Avg. Execution Time (sec) \\
\hline Trainlm & 90.57 & 0.40 \\
\hline Trainbr & 97.6 & 12.98 \\
\hline Trainscg & 79.94 & 0.15 \\
\hline Trainrp & 76.45 & 0.14 \\
\hline
\end{tabular}

Table 2 depicts that accuracy of 'Trainbr' is most excellent but requires more execution time. Taking into account both accuracy and execution time together 'Trainlm' is the most excellent training function.

Evaluation of the performance of Types of Arrhythmia Detection Using FFNN for Different Performance Functions:-The comparison of performance of FFNN using various neural network performance functions that include 'MSE','SSE','SAE, and 'MAE' is done.

Table 3: Performances for various Error function.

\begin{tabular}{|l|c|l|}
\hline \multirow{2}{*}{ Error Functions } & \multicolumn{2}{|c|}{ Feed Forward Net } \\
\cline { 2 - 3 } & Average Accuracy & Average Execution Time (sec) \\
\hline MSE & 90.57 & 1.78 \\
\hline SSE & 80.34 & 0.47 \\
\hline SAE & 82.45 & 0.55 \\
\hline MAE & 88.68 & 0.50 \\
\hline
\end{tabular}

Here average accuracy of 'MSE' is found to be 90.57 percent and the average execution time of 'MSE' performance function is almost $1.78 \mathrm{sec}$ for various number of hidden neurons.

\section{Conclusion}

In this paper, an Automated Arrhythmia Classification system is developed using statistical and spectral kurtosis features extraction and a neural network based classification tool. Total 175 ECGsamples of MIT-BIH are analyzed for classification of types of arrhythmias .By considering the performance of different $\mathrm{NN}$ architectures it's been concluded that the Feedforward neural network is the best for classification of types of arrhythmia and 'trainlm' and 'MSE' are the best training and performance functions respectively.

\section{References}

[1] MIT-BIH arrhythmia database. 3rd Ed. 1997. HarvardMIT Division of Health Science Technology, Biomedical Health Centre, Cambridge, MA, USA.

[2] http://www.physionet.org/physiobank/database/mitdb.

[3] Cuiwei Li, Chongxum Zheng and Changfeng Tai. 1995. Detection of ECG characteristic points using wavelets transforms. IEEE Transactions on Biomedical Engineering. 42(1): 21-28.

[4] B.U. Kohler, C. Henning, and R. Orglmeister, "The principles of software QRS detection”, IEEE Eng. Med. Biol. Vol. 21, pp. 42-57, 2002.

[5] M. Sabarimalai Manikandana, K.P. Somanb, "A novel method for detecting R-peaks in electrocardiogram (ECG) signals", Biomedical Signal Processing and Control 7, 2012, pp 118-128.

[6] Jaipu Pan and W. J. Tompkins, "A real time QRS detection algorithm" IEEE Transactions On Biomedical Engineering, Vol. BME-32,no. 3, March 1985 
[7] V.S.Chouhan and S.S.Mehta “Detection of QRS Complexes in 12-Lead ECG using Adaptive Quantized Threshold." International Journal of Computer Science and Network Security.28 January 2008. VOL.8.NO.1.January 2008.

[8] E. B. Mazomenos, T. Chen, "A Time-Domain Morphology and Gradient based algorithm for ECG Feature Extraction”, ICIT, IEEE, 2012, pp 117-122.

[9] P. de Chazal, M. O’Dwyer, R.B. Reilly, Automatic classification of heartbeats using ECG morphology and heartbeat interval features, IEEE Trans. Biomed. Eng. 51 (7) (2004) 1196-1206

[10] A.K.M. Fazlul Haque, Md. Hanif Ali, M. Adnan Kiber, Md. Tanvir Hasan, "Detection of small variations of ECG features using wavelet", ARPN Journal of Engineering and Applied Sciences, Vol. 4, No. 6, August 2009.

[11] Yakup Kutlu n, Damla Kuntalp, "A multi-stage automatic arrhythmia recognition and classification system", Computer in Biology and Medicine, 2011, pp 37-45

[12] Hari Mohan Rai, Anurag Trivedi, Shailja Shukla, "ECG signal processing for abnormalities detection using multi-resolution wavelet transform and artificial neural network classifier", Measurement 46 2013, pp 32383246.

[13] Issac Niwas, S.Shantha Selva Kumari, R.Sadasivam, "Artificial Neural Network based automatic cardiac abnormalities classification", Proceeding of sixth International conference on computational Intelligence and Multimedia applications, August 2005 pp.41-46.

[14] A. Dallali, A. Kachouri and M. Samet "Integration of HRV, WT and Neural Networks for ECG Arrhythias Classification.” ARPN Journal of Engineering and Applied Sciences.2011.

[15] Shivajirao M. Jadhav, Sanjay L. Nalbalwar, Ashok A. Ghatol, "Artificial neural network models based cardiac arrhythmia disease diagnosis from ECG signal data", International Journal of Computer Applications, Vol. 44, No15, April 2012.

[16] Maedeh Kiani Sarkaleh, Asadollah Shahbahrami, "Classification of ECG Arrhythmias using discrete wavelet Transform and Neural Networks", International Journal of Computer Science, Engineering and Applications (IJCSEA), Vol.2, No.1, February 2012

[17] A. T. Sayad, P. P. Halkarnikar, "Diagnosis of heart disease using neural network approach", International Journal of Advances in Science Engineering and Technology, ISSN: 2321-9009 Volume- 2, Issue-3, July2014.

[18] BOUSSAA Mohamed ECG image classification in real time based on the haar-like features and artificial neural networks The International Conference on Advanced Wireless, Information, and Communication Technologies (AWICT 2015).

[19] Siva A, Hari Sundar M, Siddharth S, Nithin M and Rajesh CB Classification of Arrhythmia using Wavelet Transform and Neural Network Model.2018

[20] Gandham Sreedevi, Bhuma Anuradha, "Classification of ECG and Identification of Cardiac Arrhythmias Using ANN”, International Journal for Research in Applied Science \& Engineering Technology (IJRASET), ISSN: 2321-9653, Vol. 6 Issue II, February 2018.

[21] Eyhab Al-Masri, "Detecting ECG Heartbeat Abnormalities using Artificial Neural Networks." 2018 IEEE International Conference on Big Data (Big Data).

[22] Yeldos A. Altay, Artem S. Kremlev "On the Use of the Statistical Methods for Biomedical Signals and Data Processing” IEEE, 2019,pp 1129-1134.

[23] R R Karhe and Dr. Sujata N Kale, "Digitization of Documented ECG signals using Image Processing ", International Journal of Engineering and Advanced Technology (IJEAT) ISSN:2249-8958, Volume-9, Issue1,October 2019 .

[24] Stalin Subbiah, Rajkumar Patro, Subbuthai P, "Feature Extraction and Classification for ECG Signal Processing based on Artificial Neural Network and Machine Learning Approach", International Conference on Inter Disciplinary Research in Engineering and Technology [ICIDRET], 2015

[25] Safa Sultan Qurraie, Rashid Ghorbani Afkhami, "ECG arrhythmia classification using time frequency distribution techniques", Korean Society of Medical and Biological Engineering and Springer, 2017.

[26] Prerna, Ram Singh,"Novel approach for prediction of heart diseases in data mining", Global Journal of Engineering Science and Researches, September 2018.

[27] Mohammed Baydoun, Lise safatly , Ossama K. Abou Hassan , Hassan Ghaziri, Ali Elhajj, Hussain Isma, "High Precision Digitization of Paper-Based ECG Records: A Step Toward Machine Learning", IEEE, VOLUME 7, 2019.

[28] Hemant Kasturiwale, Dr S N Kale, "Estimation and Analysis of Heart rate variability for Assesment of Autonomic Nervous System", Journal of Advanced Research in Dynamical and control system; SCOPUS indexed journal ,11-Special issue ,2017,page no 1-9.

[29] Seyedamin Pouriyeh, Sara Vahid, Giovanna Sanninoy, Giuseppe De Pietroy, Hamid Arabnia, "A Comprehensive Investigation and Comparison of Machine Learning Techniques in the Domain of Heart Disease”, 22nd IEEE Symposium on Computers and Communication (ISCC), 2017. 